\title{
Wissensvermittlung durch Studierende für Studierende
}

\section{Stärken und Schwächen einer spezifischen Lehrveranstaltungsform im Fachgebiet Informatik und Gesellschaft}

Thomas Herrmann, Marcel Hoffmann und Andrea Misch

Fachgebiet Informatik \& Gesellschaft,

Universität Dortmund, FB 4, LS VI, 44221 Dortmund

email: \{herrmann, hoffmann, misch\}@iug.informatik.uni-dortmund.de

Literaturstelle: Herrmann, Thomas; Hoffmann, Marcel; Misch, Andrea (1998):

Wissensvermittlung durch Stduierende für Studierende. Stärken und Schwächen einer spezifischen Lehrveranstaltung im Fachgebiet Informatik und Gesellschaft. In: Claus, Volker (Hrsg.) (1998): Informatik und Ausbildung. GI-Fachtagung 98 Informatik und Ausbildung, Stuttgart, 30. März bis 1. April 1998. Berlin et al.: Springer. S. 82-91.

\section{$1 \quad$ Einleitung}

Mit der beschleunigten Entwicklung hin zur Informationsgesellschaft gewinnt auch das Thema „Informatik und Gesellschaft“ zunehmend an Bedeutung. Dies spiegelt sich nur in wenigen Lehrveranstaltungen der Informatik wieder und dort, wo ,Informatik und Gesellschaft" explizit gelehrt wird, steht man meistens vor der Herausforderung, nicht nur die Inhalte, sondern auch die Relevanz dieser Thematik für die Informatikausbildung vermitteln zu müssen. Nach unseren Erfahrungen, fehlt es Studierenden der Informatik häufig an Vorstellungen über die Anforderungen ihres kommenden Berufslebens und dessen Vielschichtigkeit, es mangelt an Kenntnissen über die Einsatzformen der Informationstechnik und vor allem über die - meist nicht technischen Erfolgsfaktoren des Einsatzes. Das mangelnde Denken in Zusammenhängen impliziert mangelndes Problembewußtsein [Klafki 1996, S. 64] und in Folge dessen Unverständnis hinsichtlich der Relevanz einer Integration gesellschaftsorientierter Reflektion in den Methodenkanon der Informatik. Darüber hinaus richten Studierende sehr unterschiedliche, häufig unklare und in vielen Fällen wenige positive Erwartungen an das Fach Informatik und Gesellschaft. Vor diesem Hintergrund ist die Frage zu beantworten, welche Lehrveranstaltungsform für das Fachgebiet „Informatik und Gesellschaft“ zu wählen ist, wobei wir uns im folgenden auf eine Grundstudiums-Veranstaltung mit Pflichtcharakter beschränken (für eine breiter angelegte Diskussion siehe [Domeyer et. al. 1995] und [GI 1997]). Dabei kombinieren wir mittels besonderer organisatorischer Maßnahmen Eigenschaften und Vorteile verschiedener Veranstaltungsformen, wie Vorlesung, Übung, Seminar sowie fall- und empirie-orientierte Kurse. Das daraus resultie- 
rende Konzept wird hier vor dem Hintergrund von vier Jahren Erfahrung und kontinuierlicher Verbesserung dargestellt.

In Abschnitt 2 präsentieren wir die inhaltlichen Ziele der Veranstaltung und führen anschließend in Abschnitt 3 vier Themengebiete ein, die die inhaltliche Basis unseres Konzepts bilden. Die in diesen beiden Kapiteln begründete Orientierung auf die Arbeitswelt halten wir für die Lehre sozialer und gesellschaftlicher Auswirkungen von Informations- und Kommunikationstechniken aufgrund ihrer Berufsrelevanz für maßgeblich.

In Abschnitt 4 stellen wir Aufgabenstellungen vor, die nach unserer Meinung Bestandteil der Lehre von Informatik und Gesellschaft sein sollten. Dabei wird im Einzelnen erläutert, inwiefern die Aufgabenstellung die Erarbeitung der in Abschnitt 3 eingeführten Themengebiete unterstützt. Wir konzentrieren uns auf Darstellungsmethoden und auf die Befähigung der Studierenden, sich aktiv mit praktischen Problemen auseinanderzusetzen und dabei Kritik- und Argumentationsfähigkeit [Klafki 1996, S. 63] zu entwickeln.

In Abschnitt 5 werden dann verschiedene Veranstaltungsformen für die Lehre von Informatik und Gesellschaft vorgestellt und $\mathrm{zu}$ einem integrierten organisatorischen Konzept verbunden. Abschließend wird im Ausblick auf die drängendsten Probleme eingegangen.

\section{Ziele der Lehrveranstaltung}

Mit der Veranstaltung verfolgen wir eine Reihe von Zielen, aus denen sich sowohl die Inhalte als auch die eingesetzten Methoden und die Organisation der Veranstaltung ableiten lassen. Die Veranstaltung soll Studierende in die Lage versetzen, bei Diskussionen zur gesellschaftlichen Bedeutung der Informatik detailliert und vertiefend argumentieren zu können und sie befähigen, die gesellschaftliche Relevanz des im Studium vermittelten Stoffes zu beurteilen. Weiterhin soll das Bewußtsein für Problemkonstellationen geschult werden, die in unterschiedlichen Bereichen des Berufslebens auftreten können, und die Studierenden sollen lernen, Handlungsmöglichkeiten für auftretende Probleme zu identifizieren. Dabei geht es insbesondere darum zu erkennen, welche Wirkungen bzw. Folgen die Einführung und der Einsatz von Informations- und Kommunikations-Technologie (IuK-Technologien) haben kann und mit welchen Gestaltungskonzepten man auf unerwünschte Wirkungen reagieren kann. Besonders wichtig erscheint die Identifizierung von Belastungen der Benutzer sowie das Wissen um gesamtgesellschaftliche Risiken und Auswirkungen. Zusätzlich darf die Betrachtung der Veränderungen von Organisation, Kommunikation und Qualifikationsanforderungen in Betrieben nicht vernachlässigt werden. Es werden also sowohl die individuell als auch die gesellschaftlich und organisatorisch orientierten Sichtweisen gelehrt.

Die Studenten sollen in die Lage versetzt werden, eine Auswahl an Methoden und Maßnahmen treffen zu können, die in der Praxis zur Vermeidung von Problemen eingesetzt werden. Dabei ist es von besonderem Interesse, durch geeignete Konzepte und Methoden auf die spätere Kooperation und Kommunikation mit den Anwendern der IuK-Technologien vorzubereiten. So werden die Studenten befähigt, IuK-Technologien den humanen, sozialen und gesellschaftlichen Anforderungen entsprechend zu gestalten. 
In der Praxis haben wir festgestellt, daß die Studierenden die Relevanz des Faches für ihre berufliche Ausbildung als gering einschätzen. Da wir jedoch der Meinung sind, daß die Studenten für die angesprochenen Probleme sensibilisiert werden müssen, liegt ein weiteres Ziel der Veranstaltung darin, den Studenten zu vermitteln, daß es sich bei den im Rahmen der Veranstaltung anzusprechenden Themen und Problemen nicht um überflüssiges Beiwerk, sondern um Themen und Fragestellungen handelt, die für Informatiker in der beruflichen Praxis unvermeidbar und wichtig sind.

\section{Inhaltliches Konzept}

Bereits bei der Beschreibung der Ziele wurde herausgestellt, daß bei den Aufgabenstellungen von den Anforderungen der späteren Berufspraxis ausgegangen werden muß [Friedrich 1981, S. 14]. Aus diesem Grunde werden verschiedene Anwendungsfelder von IuK-Technologie herausgegriffen, die jeweils hinsichtlich unterschiedlicher Fragestellungen behandelt werden. Dabei werden Anwendungsfelder behandelt, bei denen die Einführungen von IuK-Technologien aktuell Veränderungen zeigt. In jedem Jahr werden die Anwendungsfelder auf ihre Aktualität geprüft und ggf. ersetzt. So wurde beispielsweise im vergangenen Jahr das Anwendungsfeld mobiler Kommunikation zugunsten der Betrachtung des Einsatzes neuer Medien in Informationsdiensten gestrichen. Neben diesem Feld wird in der Veranstaltung im WS 97/98 der Einsatz von IuKTechnologien im Bereich der Aus- und Weiterbildung, im Produktionsbereich und im Gesundheitswesen beleuchtet.

Die Anwendungsfelder werden unter verschiedenen Fragestellungen behandelt, die zu unterschiedlichen Themengebieten zusammengefaßt werden. Um einen Überblick über

\begin{tabular}{|l|l|l|}
\hline & Aus- und Weiterbildung & Dienstleistung und Handel \\
\hline $\begin{array}{l}\text { I: Anwendungen } \\
\text { von Informati- } \\
\text { ons- und Kom- } \\
\text { munikati- } \\
\text { onstechnik }\end{array}$ & $\begin{array}{l}\text { Vom CBT zum virtual classroom: } \\
\text { Welche Rolle spielen unterschiedliche } \\
\text { terbildung? Welche Einsatzformen und } \\
\text { Organisationskonzepte zum computer- } \\
\text { unterstützten lernen können unterschie- } \\
\text { den werden? }\end{array}$ & $\begin{array}{l}\text { Technotrends und Anwendungen im } \\
\text { Handel: } \\
\text { Welche Anwendungstypen werden } \\
\text { eingesetzt, und welche neuen Technolo- } \\
\text { gien sind gerade aktuell? }\end{array}$ \\
\hline $\begin{array}{l}\text { II: Veränderun- } \\
\text { gen von Organi- } \\
\text { sation, Kommu- } \\
\text { nikation und } \\
\text { Qualifikation }\end{array}$ & $\begin{array}{l}\text { Lernen im Wandel: } \\
\text { Werninhalte in der Informationsgesell- } \\
\text { schaft? }\end{array}$ & $\begin{array}{l}\text { Aufbauorganisation, sowie der betriebli- } \\
\text { Aelcher Veränderungen der Ablauf- und } \\
\text { chen Kommunikation ergeben sich in } \\
\text { Versicherungen bspw. durch die Einfüh- } \\
\text { rung von Telearbeit und Workflow- } \\
\text { Management? }\end{array}$ \\
\hline $\begin{array}{l}\text { III: Belastungen } \\
\text { und Software- } \\
\text { Ergonomie }\end{array}$ & $\begin{array}{l}\text { Interaktivität und Ergonomie von Lern- } \\
\text { programmen: Welche besonderen An- } \\
\text { forderungen werden an die ergonomi- } \\
\text { sche und didaktische Gestaltung von } \\
\text { Computersystemen für Aus- und Weiter- } \\
\text { bildung gerichtet? }\end{array}$ & $\begin{array}{l}\text { Belastungen durch Telearbeit: } \\
\text { Welche psychologischen und sozialen } \\
\text { Belastungen können bei Telearbeiten } \\
\text { auftreten? }\end{array}$ \\
\hline IV: Datenschutz & $\begin{array}{l}\text { Datenschutz in der Schule und Hoch- } \\
\text { schule: Welche Gesetze und Verord- } \\
\text { nungen regeln die Speicherung und } \\
\text { Verarbeitung von Daten über Schüler } \\
\text { und Lehrer? }\end{array}$ & $\begin{array}{l}\text { Datenschutz beim elektronischen Zah- } \\
\text { lungsverkehr: } \\
\text { Welche Risiken ergeben sich bei ver- } \\
\text { schiedenen Zahlungssystemen z.B. für } \\
\text { Kunden und Händler? }\end{array}$ \\
\hline
\end{tabular}

Tabelle 1: Themen und Fragestellungen 
das jeweilige Anwendungsfeld zu bekommen, werden zunächst Fragestellungen behandelt, die aktuelle Anwendungen und Risiken beschreiben. Darauf wird auf die durch die eingeführte Technologie beobachteten Veränderungen von Organisation, Kommunikation und Qualifikation eingegangen. Anschließend werden zwei Themengebiete behandelt, in deren Rahmen Mißstände aufgedeckt und Verfahren erarbeitet werden, wie diese Mißstände in Zukunft bereits im Vorfeld umgangen werden können. Dabei wird einerseits auf die Belastung durch Computerarbeit und Anforderungen an benutzerfreundliche Software-Gestaltung eingegangen. Andererseits werden wesentliche Aspekte von Problemen des Datenschutzes und der Datensicherheit behandelt. Wie nachfolgend erläutert wird, liegt der Schwerpunkt der Themengebiete und ihrer Fragestellungen dabei weniger auf den Artefakten der IuK-Technik selbst als vielmehr auf der Betrachtung ihres Anwendungskontextes [vgl. dazu Dahlbom\&Mathiassen 1997, S. 86]. Als Beispiel zeigt Tabelle 1 einige Fragestellungen, mit denen Themengebiete auf dem Anwendungsfeld der Aus- und Weiterbildung bzw. im Bereich Dienstleistungen und Handel diskutiert wurden.

\subsection{Beschreibungsmöglichkeiten für Anwendungen und Risiken}

Um Wirkungen oder Folgen von IuK-Technologien diskutieren zu können, aber auch um Vor- und Nachteile unterschiedlicher Gestaltungsvarianten zu beurteilen, werden modellhafte Beschreibungen von IuK-Technologien benötigt (vgl. Kap. 4.1). Mit Hilfe der Modellierungsmethoden sollen Studierende erlernen, eine Anwendung von Informations- und Kommunikationstechnik aus verschiedenen Blickwinkeln zu betrachten. Dabei üben sie im Einzelnen,

- eingesetzte Hard- und Software zu differenzieren und die Architektur der Anwendung mit Hilfe von Architekturmodellen zu beschreiben,

- den Zweck des Systems mit den Interessen der Beteiligten zu vergleichen, indem man versucht, soziotechnische Systeme zu modellieren,

- mit Ablaufdiagrammen sowie Daten- und Organisationsmodellen den Einsatz des Systems in Arbeitsprozessen und den Beitrag zur Verarbeitung von Informationen und Daten in einer Organisation darzustellen sowie

- die dahinter stehende Art und Weise der Entwicklung und Einführung von Software anhand von Vorgehensmodellen zu hinterfragen.

Die Beschreibung von Anwendungen und Risiken bildet die Grundlage für die Untersuchung des Einsatzes von IuK-Technologie unter speziellen Fragestellungen, die in den folgenden drei Abschnitten erläutert werden.

\subsection{Veränderung der Organisation, Kommunikation und Qualifikation}

Als zweites Themengebiet behandelt die Veranstaltung Veränderungen von Organisation, Kommunikation und Qualifikation als Folge des Einsatzes von Informations- und Kommunikationstechnik. Dabei geht es insbesondere darum, die wechselseitigen Einflüsse neuer Techniken und innovativer Organisationsformen sowie der Kommunikation und der Qualifikation der Benutzer hervorzuheben. Die Fragestellungen lauten beispielsweise: Wie verändern sich Unternehmensformen und die Aufbau- und Ablauforganisation? Wie verändern sich Kommunikationsinhalte und -stile? Welche neuen Qualifikationen erfordert der Umgang mit Informations- und Kommunikationstechnik? 


\subsection{Belastung durch Computerarbeit und Anforderungen an benutzer- freundliche Software-Gestaltung}

Belastungen der Benutzer sind in der Praxis oft eine wesentliches Hindernis für die Akzeptanz und den Erfolg neuer Technologien. Dabei verstehen wir unter Belastungen Hindernisse oder Erschwerungen des (Arbeits-) Handelns, die mit dem Arbeitsmittel oder der Organisation seines Einsatzes verbunden sind [s. z.B.: Frese\&Brodbeck 1989], z.B. durch mangelnde Software-Ergonomie oder durch technisch bedingte Einschränkungen von Handlungs- und Gestaltungsspielräumen. Solche Belastungen können Beeinträchtigungen oder Schädigungen der Benutzer hervorrufen oder der Entfaltung und Weiterentwicklung von Fähigkeiten entgegenstehen. Das Thema SoftwareErgonomie gibt Gelegenheit, auf derzeit aktuelle Normierungsfragen auf diesem Gebiet einzugehen, Mißstände aufzudecken und ihre Relevanz für das spätere berufliche Wirken von Informatikern zu verdeutlichen.

\subsection{Wesentliche Aspekte von Problemen des Datenschutzes und der Datensicherheit}

Neben der Software-Ergonomie stellt der Datenschutz ein weiteres klassisches Thema von Informatik und Gesellschaft dar. Dabei geht es um Regelungen und Konfliktpotentiale, die bei der Gestaltung von Informations- und Kommunikationssystemen berücksichtigt werden müssen. Die Bearbeitung dieses Themengebiets führt die Teilnehmer in wesentliche Prinzipien des Datenschutzes und rechtliche Rahmenbedingungen ein. Außerdem lernen die Teilnehmer Konfliktpotentiale an konkreten Beispielen für Verstöße gegen die Gesetzgebung, für Mißbrauch personenbezogener Daten und für Regelungen von Steitfällen im betrieblichen Kontext kennen. Ferner wird unter dem Aspekt Datensicherheit auf Risiken des Einsatzen von Informationssystemen eingegangen [Roßnagel 1989]. Insgesamt wird mit dieser Themenkombination das Ziel verfolgt, daß Studierende zwischen unerwünschtem und gewünschtem sowie zwischen mißbräuchlichem und regelgerechtem Gebrauch von Informationssystemen unterscheiden lernen.

\section{Aufgabenstellungen und Methoden zur Vermittlung der Inhalte}

Wie bereits erwähnt fordert der klassische Vorlesungsbetrieb die Studierenden nach unserer Einschätzung nicht ausreichend zur aktiven Mitarbeit heraus. Statt dessen sollten die Studierenden unterschiedliche Aufgabenstellungen bearbeiten, die im Folgenden erläutert werden.

\subsection{Modellierung}

Es fällt auf, daß die in der Informatik verbreiteten Beschreibungsmethoden wie der Entity-Relationship Ansatz, die Strukturierte Analyse oder Petri-Netze keine ausreichenden Möglichkeiten zur Modellierung sozial und gesellschaftlich relevanter Dimensionen beinhalten. Viele Modellierungsmethoden beschränken sich auf die Modellierung technischer Aspekte wie Datenflüsse oder Schnittstellenoperationen. Für die Abschätzung von Risiken werden jedoch Modellierungsmethoden benötigt, mit denen beispielsweise auch Belastungen der Benutzer, Qualitäten von Arbeitsaufgaben oder der 
Mißbrauch personenbezogener Daten modelliert werden können [zu Anforderungen an entsprechende Modellierungsmethoden siehe Herrmann et al. 1997].

Im Rahmen der Grundveranstaltung werden daher verschiedene Modellierungsmethoden kombiniert sowie deren Einsatz für die Identifikation und Darstellung von Problemund Konfliktfeldern geübt. Dabei dient die Modellierung verschiedener Anwendungen von IuK-Technologie als Einstieg in die Betrachtung der Arbeitswelt und dem Kennenlernen besonders relevanter Anwendungsfelder (vgl. Kapitel 3).

\subsection{Empirie}

Damit die Studenten spätere Problemkonstellationen im Berufsleben erkennen und einschätzen können, scheint es sinnvoll, die Lehrveranstaltungen mit starkem Praxisbezug zu versehen. Um dies zu gewährleisten, werden die Studenten angehalten, in dem ihnen zugeteilten Anwendungsfeld empirisch zu arbeiten und so konkret Wirkungen und Folgen des Einsatzes von IuK-Technologien zu beobachten. Durch Gespräche mit Mitarbeitern können beispielsweise konkrete Belastungen von Nutzern oder die Veränderung der Organisation, Kommunikation oder Qualifikationsanforderungen kennengelernt werden. Da vielen Teilnehmern der Veranstaltung bei den Gesprächen in den besuchten Firmen erstmals Probleme des Einsatzes von IuK-Technologien bewußt werden, liefert die Empirie einen großen Beitrag, um die Relevanz der Themen und Fragestellungen herauszustellen. Dabei scheint es sinnvoll, daß Studierende ihr empirisches Vorgehen im Sinne einer Fallstudie betreiben, bei der es auch darum geht, Verbesserungspotentiale auszuloten und entsprechende Verbesserungsvorschläge $\mathrm{zu}$ skizzieren.

\subsection{Literatur}

$\mathrm{Zu}$ den verschiedenen Themen existieren wissenschaftliche Arbeiten und weitere Literatur, die Hintergrundwissen vermitteln (s. z.B. zum Thema Anwendung bei Aus- und Weiterbildung [Schulz-Zander 1995]) und die zur umfassenden Betrachtung der Themen notwendig scheinen. So ist es beispielsweise besonders für den Bereich Datenschutz unerläßlich, sich zunächst mit den speziellen Gesetzen vertraut zu machen, bevor man für einen konkret beobachteten Fall Mißbrauch identifizieren und Handlungsmaßnahmen vorgeschlagen werden können.

Die Betrachtung der Literatur empfiehlt sich überdies, um Grenzen der Empirie entgegenzuwirken. Bei ausschließlich empirischen Arbeiten würde die Forschung auf dem Gebiet von Informatik und Gesellschaft vernachlässigt werden und Entwicklungen in anderen Ländern nur schwerlich Beachtung finden. Auch scheint die Beachtung gesamtgesellschaftlicher Risiken und Auswirkungen für empirische Untersuchungen seitens der Studenten unmöglich, da Zeit und Mittel unzureichend sind. Die Betrachtung aktueller Forschungsberichte und internationaler Studien scheint deshalb ebenso unerläßlich wie das Beachten von Langzeitstudien oder Feldversuchen zu gesamtgesellschaftlichen Risiken und Auswirkungen. Dabei ist es ein wichtiges Lernziel, daß Studierende zwischen wissenschaftlicher Literatur und der Analyse und Auswertung von Dokumenten unterscheiden lernen, wobei letztere keine wissenschaftlichen Erkenntnisse, sondern eher Meinungsäußerungen enthalten. Die Vermischung von beidem ist gerade im Fachgebiet Informatik und Gesellschaft naheliegend. Die Schulung entspre- 
chender Differenzierungsfähigkeiten ist zur Zeit um so wichtiger, da viele Studierende beginnen, recht unkritisch Informationen aus dem WWW zu übernehmen und als Referenzen in ihren Seminararbeiten verwenden, ohne den unterschiedlichen Stellenwert ihrer Quellen zu verdeutlichen.

\subsection{Vorträge}

Die Inhalte aller vier Themen, die durch Empirie, Literaturrecherche und Modellierung erarbeitet wurden, werden von den Studierenden mittels foliengestütztem Vortrag mit zugehörigem Thesenpapier an die anderen Studenten weitergegeben. So wird gewährleistet, daß sie sich als Vortragende durch Vorbereitung des Vortrags aktiv mit dem Thema auseinandersetzen. Zudem lernen sie, selbst Zusammenhänge darzustellen und vor anderen zu präsentieren. Referate werden zu Übungszwecken in kleinen Gruppen gehalten und nach einer Überarbeitung in großen Gruppen präsentiert (s. Kap. 5). Hierdurch haben die Studenten als Hörer Gelegenheit, auch andere Anwendungsfelder als das selbst Erarbeitete kennenzulernen.

\subsection{Diskussion}

Auf Grundlage der studentischen Vorträge und der ausgearbeiteten Thesenpapiere folgen Diskussionen, die von einem Tutor (Übungsgruppenleiter) oder dem Dozenten selbst geleitet werden. Dabei lernen die Studenten, den eigenen Standpunkt darzustellen und zu vertreten sowie andere Standpunkte aufzunehmen und darauf einzugehen. Diese Diskussionen schulen insbesondere die Fähigkeit, über Probleme zu sprechen und bereiten so auf die spätere Kommunikation mit Benutzern vor. Außerdem dienen die Diskussionen der Schulung detaillierter und vertiefender Argumentation und Reflektion, die bei späteren Diskussionen zur gesellschaftlichen Bedeutung der Informatik benötigt werden. Die Moderation der Diskussion verfolgt das Ziel, Sachaussagen der Vortragenden notfalls zu korrigieren bzw. zu ergänzen, Argumentationslinien und ihre Interessenhintergründe transparent zu machen, die Vertiefung der Argumentation herauszufordern und damit auch Wege aufzuzeigen, wie die systematische Auseinandersetzung mit einem Thema strukturiert werden kann.

\section{Organisatorisches Konzept}

Die bisherige Erfahrung zeigt, daß sich die klassische Kombination von Vorlesungseinheiten und parallel zu bearbeitenden Übungsaufgaben zur Erreichung der dargestellten Ziele der Veranstaltung wenig eignet. Vielversprechender erscheint es, wenn sich Studierende aktiv mit dem Stoff auseinandersetzen, indem sie selbst Thesen ausarbeiten und diese durch Kurzreferate zur Diskussion stellen. Aufbauend auf der Annahme, daß Grundlagen am besten per Vorlesung, Anwendungen jedoch als komplexe Beispiele in Diskussionen vermittelt werden sollten, wurde ein Organisationskonzept entwikkelt, das den klassischen Vorlesungsbetrieb mit seminarähnlichen Übungsgruppen verbindet und als zusätzliche Veranstaltungsform regelmäßig stattfindende große Übungsgruppe sogenannte Plena integriert, die ab der 10. Woche die Vorträge des Dozenten ersetzen. Im folgenden werden die Bestandteile des Organisationskonzeptes einzeln vorgestellt und begründet. Abbildung 1 gibt einen Überblick über das Organisationskonzept . Die Teilnehmerzahlen beziehen sich auf das WS 1997/98: 


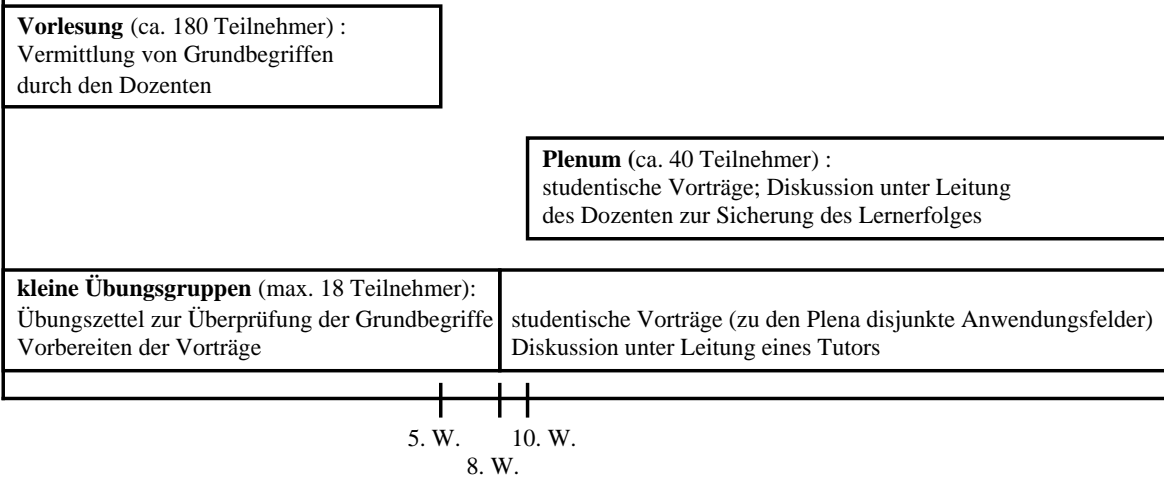

Abbildung 1: Das Organisationskonzept

\subsection{Vorlesung, Kompendium und Übungsaufgaben}

$\mathrm{Zu}$ Beginn der Veranstaltung werden in 5 Vorlesungseinheiten Grundlagenkenntnisse über die Fragestellungen und die Methoden des Fachgebietes Informatik und Gesellschaft vermittelt. Diese Einheiten befassen sich jeweils mit den in Abschnitt 3 vorgestellten Themengebieten. Begleitet werden die Vorträge durch Übungsaufgaben, die von den Leitern der Übungsgruppen korrigiert und in den Übungsgruppen besprochen werden. Die Bearbeitung der Übungsaufgaben wird durch Hinweise auf ein Lehrbuch [Friedrich et al. 1995] und ein die Veranstaltung begleitendes Kompendium unterstützt. Vorträge, begleitendes Material und Übungsaufgaben erlauben es den Studierenden, sich in grundlegende Fragestellung einzuarbeiten und sich in empirischen Methoden und Techniken der Modellierung und Gestaltung von Anwendungen von IuKTechnologien zu üben.

\subsection{Kleine Übungsgruppen mit Seminarcharakter}

Parallel zu den letzten Vorträgen des Dozenten beginnen Studierende, Fragestellungen aus einem Anwendungsfeld und einem Themengebiet (vgl. Abschnitt 3) zu bearbeiten. Dabei werden sämtliche in Abschnitt 4 erläuterten Aufgabenstellungen abgedeckt. Insbesondere sind die Studierenden gefordert, empirisch $\mathrm{zu}$ arbeiten, indem sie beispielsweise Interviews mit Firmenvertretern führen oder konkrete Softwaresysteme erproben und bewerten, und ihre Ergebnisse in Modellen systematisch darzustellen. Parallel dazu bereiten sie ihre Fragestellung durch Literaturrecherche auch von der theoretischen Seite auf. Ein Handapparat erleichtert den Einstieg in die Literaturrecherche. Besonders beauftragte Ansprechpartner beraten die Studierenden bei der Vorbereitung und Durchführung der empirischen Arbeit.

Ab der 8. Woche werden in den Übungsgruppen keine Übungsaufgaben mehr besprochen, sondern nur noch Vorträge gehalten und moderierte Diskussionen geführt. Dabei behandelt jede Übungsgruppe schwerpunktmäßig zwei bis drei Anwendungsfelder. Die Referate werden als Gruppenarbeit, von zwei bis drei Studierenden vorbereitet, die Diskussion der Vorträge wird vom Übungsgruppenleiter moderiert. Die Übungsgruppe gewinnt dadurch den Charakter einer Seminarveranstaltung. Im Laufe des Semesters referieren alle Teilnehmer in den Übungsgruppen. Einige übernehmen zwei Themen andere vertiefen ein Thema und referieren später noch einmal in einem Plenum. 
Die Ersetzung der Übungsaufgaben durch Referate eröffnet den Studierenden Spielräume, Inhalte und Fragestellungen der Veranstaltung mitzubestimmen. Außerdem entwickelt die Veranstaltung durch die empirischen Arbeiten und Berichte der Studierenden einen starken Praxisbezug, der von einem Dozenten allein nicht entsprechend vielseitig und anschaulich hergestellt werden könnte.

\subsection{Große Übungsgruppe (Plenum)}

Obgleich die Fokusierung der Veranstaltung auf das Erarbeiten von Praxisberichten sich im Hinblick auf die Motivation positiv auswirkt, birgt die Verlagerung wesentlicher Lehrinhalte in die Verantwortung der Studierenden auch Risiken im Hinblick auf das Lernergebnis. Insbesondere spielt die Qualität der Vorträge in den Übungsgruppen eine ganz wesentliche Rolle für den Erfolg der Veranstaltung insgesamt.

Um zu vermeiden, daß in weniger guten Vorträgen wesentliche Lerninhalte unzureichend dargestellt werden und dadurch Ziele der Veranstaltung verfehlt werden, wird der im vorangegangenen Abschnitt erläuterte Übungsbetrieb ab der 10. Veranstaltungswoche durch große Übungsgruppen, sogenannten Plena ergänzt, die dann an Stelle der Vorlesung treten. $\mathrm{Zu}$ einem Plenum treffen sich einmal pro Woche Studierende aus zwei bis drei Übungsgruppen und hören Referate, die zuvor in anderen Übungsgruppen geprobt wurden. In diesen Plena übernimmt der Dozent die Rolle des Diskussionsleiters und versucht, durch seine fachlichen Kommentare verschiedene Perspektiven und wissenschaftliche Aspekte in die Diskussion einzubringen. Die Kombination großer und kleiner Übungsgruppen ermöglicht den Studierenden das Kennenlernen vieler Anwendungsfelder und sichert gleichzeitig den Lernerfolg insgesamt.

\section{Zusammenfassung und Ausblick}

Trotz der stärkeren Praxisorientiertung der Veranstaltung und der in vielen Fallstudien offenkundig werdenden Probleme, Konflikte und Ungereimtheiten des Informationstechnikeinsatzes, ist die Diskussionsbereitschaft der Studierenden im Durchschnitt relativ gering und konzentriert sich auf eine kleine Zahl (ca. 20 - 25\%) aktiver Teilnehmer. Grund hierfür ist nach unserer Vermutung zum einen, daß Studierende sich nicht auf die Vorstellung einlassen, daß die von ihnen untersuchten Themen und Fälle zu einem großen Teil Aspekte ihres künftigen Berufslebens präsentieren. Dies mag darauf beruhen, daß ihre Ansprechpartner in den empirischen Feldern häufig NichtInformatiker sind. Hier kann es eine Abhilfe sein, künftig zu fordern, daß pro empirischer Untersuchung die Sichtweise eines Informatikers mit der eines NichtInformatikers verglichen wird.

Weiterhin erweist es sich als Problem, daß es nahezu unmöglich ist, den Zugewinn von Diskussions- und Argumentationsvermögen zum Gegenstand üblicher Leistungsüberprüfungen zu machen. Diese sind aber für viele Studierende ein verständlicher Hintergrund ihrer Prioritätensetzung, da auch sie versuchen, mit den Ressourcen ihrer Leistungsfähigkeiten wirtschaftlich umzugehen. Es sind geeignete Aufgabenstellungen zu finden, die argumenativstrukturiertes Reflektieren fordern und fördern. 
Drittens scheinen viele Informatiker ein systematisches Defizit bzgl. der argumentativen Durchdringung von Sachverhalten zu haben - dies zeigt sich auch, wenn es darum geht, rein technische Sachverhalte zu ergründen. Es besteht die Tendenz, allzu vorschnell mit der erst besten Erklärung bzw. Lösung oder eben mit dem erst besten Argument zufrieden zu sein. Hier bedarf es einer gemeinsamen Anstrengung aller Fachvertreter der Informatik, um ein tiefergreifendes Analyse- und Reflektionsvermögen der Studierenden zu fördern.

\section{Literatur}

[Dahlbom\&Mathiassen 1997]

Dahlbom, Bo; Mathiassen, Lars (1997): The Future of Our Profession. There's more to being a good engineer than a high level of technical competence. In: Communications of the ACM Vol. 40, No. 6, June 1997. S. 80-89.

[Domeyer et al.]

Domeyer, Volker; Grusdat, Matthias; Schmidthalks, Fiedemann; Wildt, Johannes: Didaktik von „Informatik und Gesellschaft“ in [Friedrich et al. 1995]. S. 315-321.

[Frese\&Brodbeck 1989]

Frese, Michael; Brodbeck, Felix C.: Computer in Büro und Verwaltung. Heidelberg et al.: Springer Verlag. 1989.

[Friedrich 1981]

Friedrich, Jürgen: "Informatik und Gesellschaft" als Gegenstand des InformatikStudiums. 1981.

[Friedrich et al. 1995]

Friedrich, Jürgen; Herrmann, Thomas; Peschke, Max; Rolf, Arno (Hrsg.): Informatik und Gesellschaft. Heidelberg, Spektrum Akademischer Verlag. 1995.

[GI 1997]

Arbeitskreis „Neue Lehrinhalte und Veranstaltungsformen im Informatikstudium“ im Fachausschuß 7.1 der Gesellschaft für Informatik e.V. (GI): Ergänzende Empfehlung der Gesellschaft für Informatik Lehrinhalte und Veranstaltungsformen im Informatikstudium. In: Informatik Spektrum Vol 20. Heft 5/1997. S. 302-306.

[Herrmann et al. 1997]

Herrmann, Th.; Hoffmann, M.; Loser, K.-U.: Modellierungsnotationen für prospektive, gestaltungsorientierte Technikfolgenforschung. In: Paul, H.: Modellierung von Aufbau- und Ablauforganisation: von der Technozentrik zur Anthropozentrik. Projektbericht des Instituts Arbeit und Technik. 02-97. S. 33-46.

[Klafki 1996]

Klafki, W.: Neue Studien zur Bildungstheorie und Didaktik. 5. Auflage. Weinheim, Basel: Beltz Verlag. 1996.

[Roßnagel 1989]: Roßnagel, Alexander; Wedde, Peter; Hammer, Volker; Pordesch, Ulrich: Die Verletzlichkeit der Informationsgesellschaft. Opladen: Westdeutscher Verlag. 1989.

[Schulz-Zander 1995]: Schulz-Zander, Renate: Informationstechnologien im Bildungsbereich. In: [Friedrich et al. 1995]. S. 114-122. 